\title{
Analysis of residuals in daily solar radiation time series
}

\begin{abstract}
The Box-Jenkins approach is applied to daily solar radiation data from four different locations in Malaysia. The deterministic annual component is obtained by Fourier analysis. The stochastic component of the time series is fitted to three models, ARMA $(1,0)$, ARMA $(2,0)$ and ARMA $(1,1)$. Random shocks from these models are tested by Box-Pierce statistic and Ljung-Box for whiteness of residuals. Skewness and kurtosis coefficients are tested for normality.
\end{abstract}

Keyword: Solar radiation; Time series; Residuals 